\title{
CORRESPONDENCE
}

\section{Predicting survival in malignant mesothelioma}

\section{To the Editors:}

The model proposed by MUSK et al. [1] to predict survival from diagnosis for patients with malignant mesothelioma has some anomalies which need to be addressed. First, there are two typographical errors. The model claims to predict "survival in newly diagnosed cases of MM (in weeks)" whereas it actually predicts the survival probability. Survival time after diagnosis (in months) is part of the model formula, so it cannot also be the outcome. Also, the value -1.04 in the model equation should be replaced by -0.97 (N. de Klerk, Schools of Population Health and Medicine and Pharmacology, Perth, Australia; personal communication).

Secondly, the terms involving age (in yrs) arouse concern because they are so complicated, i.e. $+0.004(\text { age } / 10)^{3}-0.002$ (age/ $10)^{3} \mathrm{Ln}($ age/10), and they do not reflect a monotonic decrease in survival with age. They are not consistent with the statement in the abstract that "Survival was inversely related to age...", which appears to be based on median survival times over four unequal age cohorts from $20-49$ yrs to $\geqslant 75$ yrs. There was no survival graph showing the effect of age at diagnosis, and age was not included in table 2 which showed the Cox regressionbased hazard ratios of all the other prognostic factors. The agerelated terms in the model mean that patients in their early $50 \mathrm{~s}$ are predicted to have longer survival than patients in their 40 s or 70s. This is implausible.

Finally, the coefficients for mesothelioma in the left or right pleura are -0.15 and -0.16 , respectively, which mean the predicted survival is worse for both these groups of patients than for the unknown number of patients in the reference group with unspecified pleural mesothelioma. This is also implausible. In table 2 the hazard ratios for both left and right pleura were not significantly different from zero (p-values 0.14 and 0.07, respectively), which implies they could be omitted from the model.

As this model may be used to predict survival in legal compensation cases, it needs to be able to withstand rigorous scrutiny. A revised model, which addresses these anomalies, would be useful.

Jennifer G. Smith

Plain Statistics Pty Ltd, Horsham, Australia.

Correspondence: J.G Smith, Plain Statistics Pty Ltd, PO Box 734, Horsham, Victoria 3402, Australia. E-mail: jgsmith@ plainstatistics.com

Statement of Interest: None declared.

\section{REFERENCES}

1 Musk AW, Olsen N, Alfonso $\mathrm{H}$, et al. Predicting survival in malignant mesothelioma. Eur Respir J 2011; 38: 1420-1424.

DOI: 10.1183/09031936.00088912 